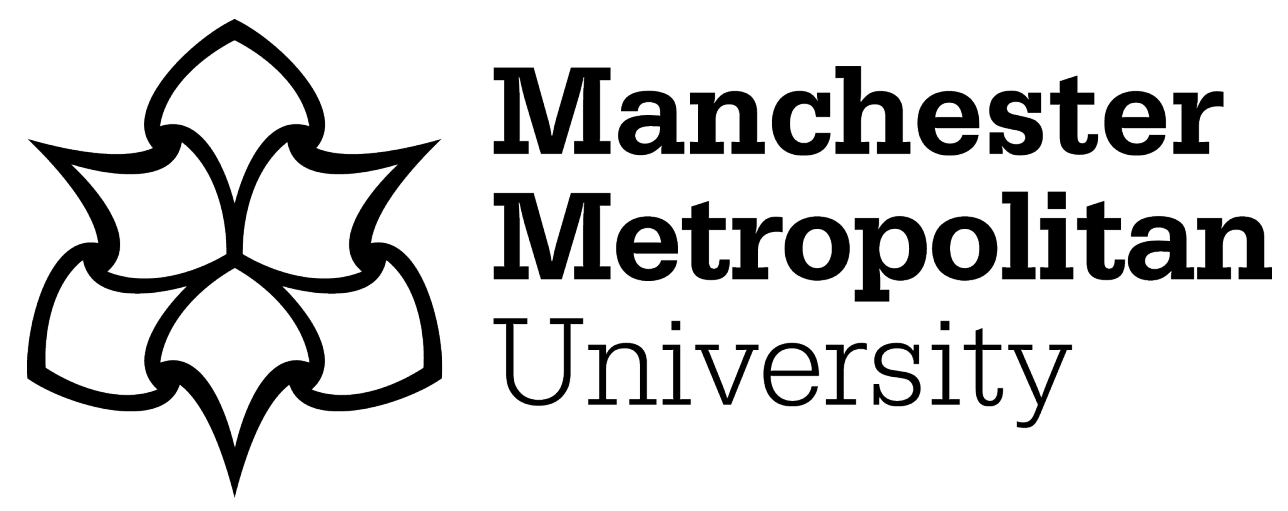

Blackhurst, E and Salmon, N ORCID logoORCID: https://orcid.org/00000003-4786-7130 (2019) Full polarimetric radar for concealed weapons detection: Experimental determination and simulation of the Huynen target parameters for the human torso. In: Proceedings Volume 11164, Millimetre Wave and Terahertz Sensors and Technology XII (SPIE Security + Defence, 2019, 09 September 2019 - 12 September 2019, Strasbourg, France.

Downloaded from: https://e-space.mmu.ac.uk/624993/

Publisher: The International Society for Optical Engineering

DOI: https://doi.org/10.1117/12.2547833

Please cite the published version 


\title{
Full polarimetric radar for concealed weapons detection: Experimental determination and simulation of the Huynen target parameters for the human torso
}

\author{
Eddie Blackhurst ${ }^{\mathrm{a})}$, Neil Salmon ${ }^{\mathrm{b})}$, School of Physics and Astronomy, a) The University of \\ Manchester, Jodrell Bank, SK11 9DL. b) Faculty of Science and Engineering, Manchester \\ Metropolitan University, All Saints, Oxford Road, M15 6BH.
}

\begin{abstract}
The measurements of the human torso for two individuals are presented via the generation of the Huynen polarisation fork technique and plotted on the Poincaré sphere, to ascertain characteristics that could be used to remove the effects of the torso when concealed weapons are placed against it. Measurements are taken with a frequency modulated continuous wave (FMCW) mono-static millimetre wave full polarimetric radar, operating at k-band (18 to $26 \mathrm{GHz}$ ). The system has been designed to explore the feasibility of using full polarimetry for the detection of concealed weapons, and person borne improvised explosive devices (PBIED). The philosophy of this scheme is a means to extract the maximum information content from a target which is in the nominally single spatial pixel (sometimes sub-pixel) configuration of stand-off (tens of metres) and crowd surveillance scenarios. The radar comprises a vector network analyser (VNA) and an orthomode transducer.
\end{abstract}

Keywords: Concealed weapons detection, FMCW radar, Mono-static radar, full polarimetry, millimetre wave radar, non-imaging sensor, Radar calibration, Huynen polarization fork.

\section{INTRODUCTION}

The microwave and millimetre wave band are ideally suited to the recognition of concealed weapons on persons as clothing is almost completely transparent in this frequency band. The great opportunity here is to exploit the huge body of knowledge on techniques developed by the defence and remote sensing for polarimetric radar over the past 50-years. The work presented in this paper represents the latest implementation of these techniques, following on from an initial look at the Huynen Target parameters and the Huynen Polarisation Fork [1] to extract information associated with the concealed weapons. This paper concentrates on understanding the response of the human torso by itself to full polarimetric radar.

\section{METHOD}

The radar presented in this paper is of the monostatic non imaging type based around Sinclair matrices generated from measurements made with a Keysite PNAX VNA, with a swept frequency from $18 \mathrm{GHz}$ to $26 \mathrm{GHz}$ (K band) divided in to 801 spectral increments. The radar is of the frequency modulated carrier wave (FMCW) type generating a Sinclair matrix for each spectral increment across the band. A block diagram of the radar can be seen in Figure 1. The horn antenna is of the conical corrugated type and the ortho-mode transducer (OMT) is of the linear turnstile type with isolation better than $-35 \mathrm{db}$ between orthogonal polarisations [2]. Both ports of the VNA are used and with the OMT connected port 1 represents the horizontal linear polarisation whilst port 2 represents the vertical. Measurements are coherent using both phase and magnitude. The VNA generates scattering parameters which when connected as in Figure 1 directly represent the Sinclair matrix produced by reflection from a target (Eq 1.).

$$
S=\left[\begin{array}{ll}
S_{11} & S_{12} \\
S_{21} & S_{22}
\end{array}\right]=\left[\begin{array}{ll}
S_{H H} & S_{H V} \\
S_{V H} & S_{V V}
\end{array}\right]
$$




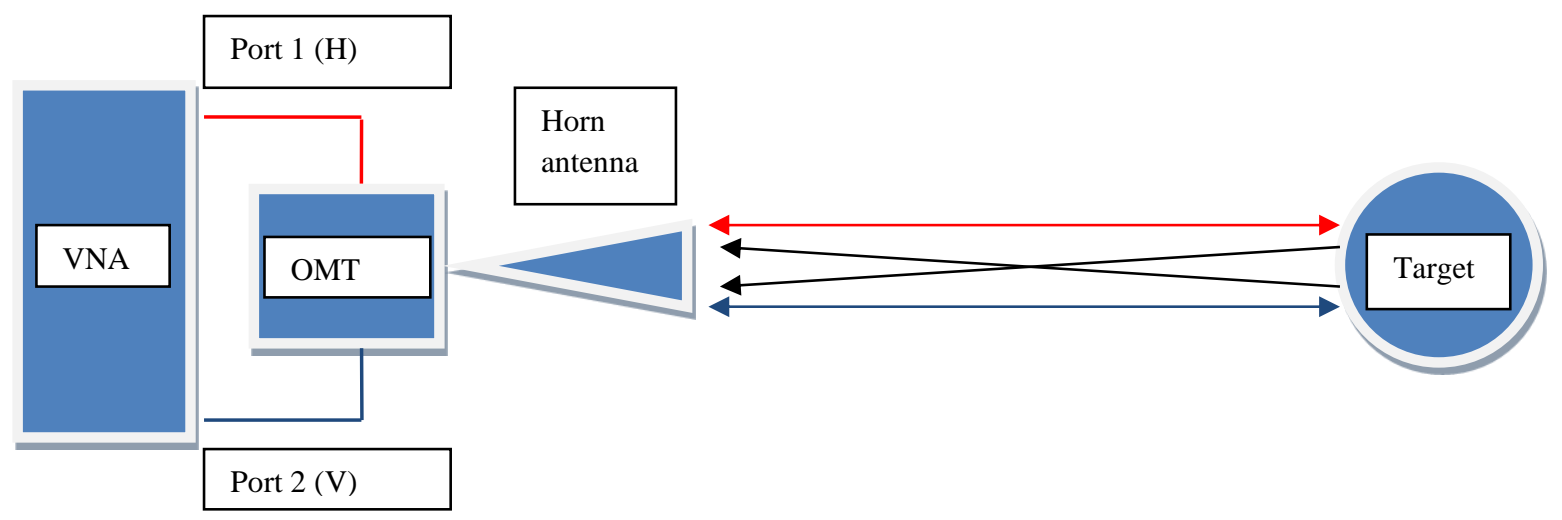

Figure $1 \mathrm{~A}$ schematic of the full polarimetric monostatic FMCW radar.

Calibration of the system is made by making a Sinclair matrix measurement of a plane reflector, a dihedral reflector orientated at $45^{\circ}$ and a background measurement (the scene with the target removed). This is then used to determine the calibrated Sinclair matrix by using Eq. 2.

$$
\text { Calibrated Target }=\frac{(\text { Target }- \text { RAM })}{\left(\text { DH } 45^{\circ}-\text { RAM }\right)+(\text { Plate }- \text { RAM })}
$$

This calibrates the phase and amplitude of the Sinclair matrices (at each frequency). This calibration process removes the effects of dispersion in the orthomode transducer and the antenna, leaving just the response from the antenna aperture out and back to the subject and its surrounding.
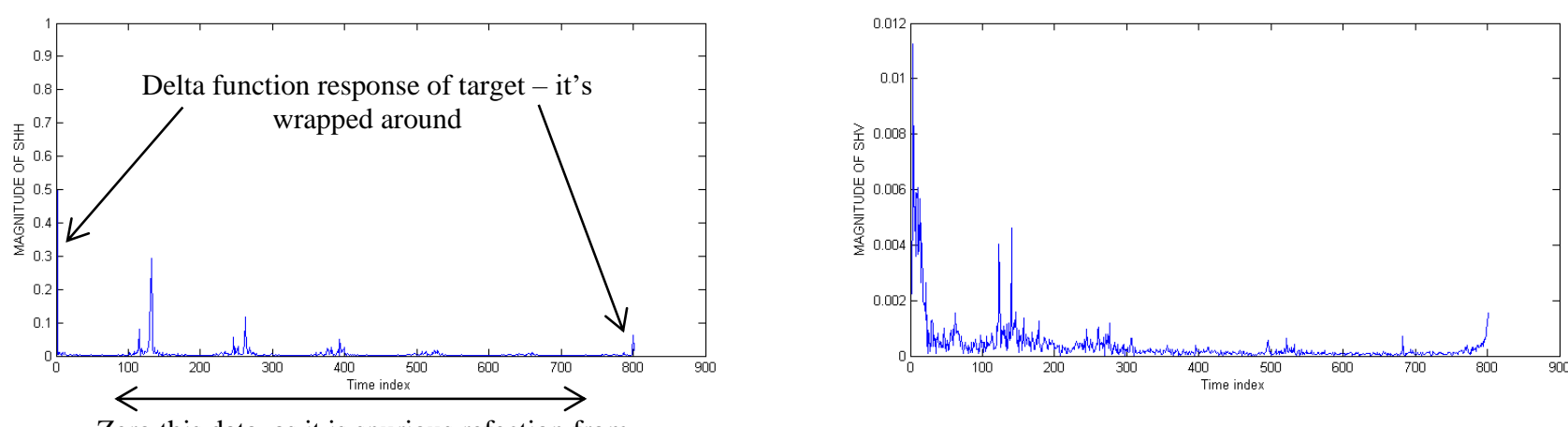

Zero this data, as it is spurious refection from
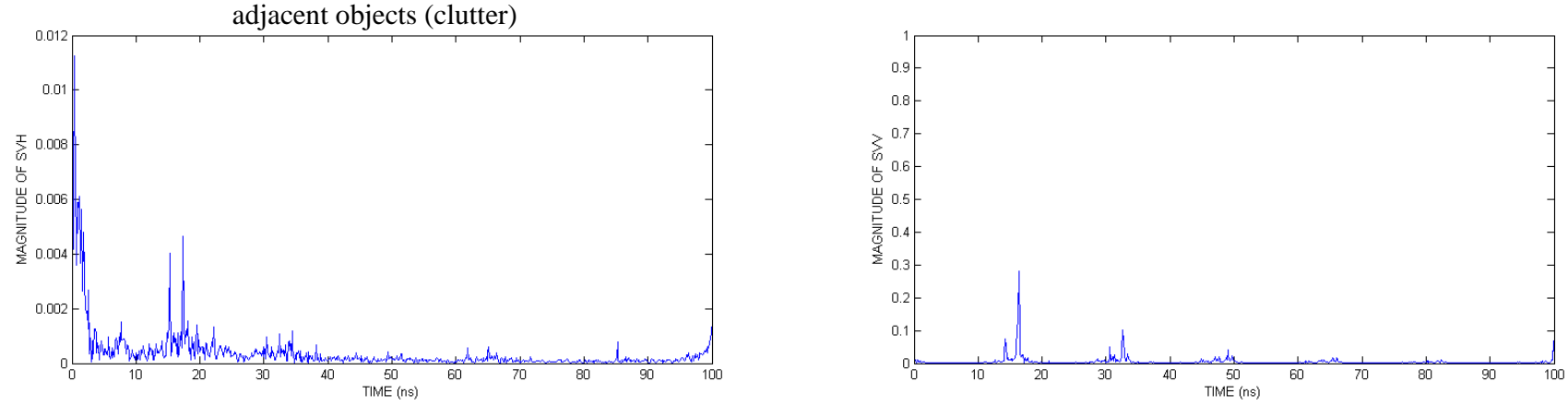

Figure 2. Calibrated Sinclair matrices (clockwise from the top left: $\mathrm{S}_{\mathrm{HH}}, \mathrm{S}_{\mathrm{HV}}, \mathrm{S}_{\mathrm{VH}}, \mathrm{S}_{\mathrm{Vv}}$ ) representing the time domain (delta function) response of a flat plate target, illustrating reflection from the target and other objects (clutter) around the target which can be removed by range gating, before returning to the frequency domain for further processing. 
Clutter generated by reflections from objects in the scene other than the subject can be examined by making a Fourier transform of the calibrated Sinclair matrices. This creates the time domain (or delta function) response of the scene, the magnitude of which is shown in Figure 2. This reveals how reflections would arise from a short (delta function) being sent to the scene. In the experiment no delta function is transmitted, only excitations at a range of frequencies $18 \mathrm{GHz}$ to $26 \mathrm{GHz}$, stepped linearly in frequency in steps of (26-18) $\mathrm{GHz} / 800 \sim 10 \mathrm{MHz}$. The $\mathrm{x}$-axis is displayed both as index number and time in nanoseconds. The delta function response from the target, in this case a flat plate, can be seen as a broadened short spike at the start and end of the trace. (The trace wraps around.) If desirable this trace can be shifted in time by applying a linear phase shift before the Fourier transform process. Also seen in the delta function response are reflections from other objects surrounding the flat plate, including multiple reflection from the table on which the horn antenna is placed. All objects that are not part of the target are clutter, and their response can be removed from the trace by simply assigning those data elements of the array to zero. This effectively range gates the data, effectively accepting only reflection from the target and its immediate vicinity. This refines the delta function, effectively removing the clutter from the data, enabling a much cleaner response from the target to be processed. This refined delta function is then Fourier transformed back to the frequency domain for further algorithmic processing.

\section{POLARISATION ON THE POINCARÉ SPHERE}

Graphical representation of any polarization state can be defined as a point $\mathrm{P}$ when plotted on the Poincaré sphere (Figure 3a) [3]. This unit radius sphere was developed by Poincaré in 1892 to represent the polarization states of polarized light. The zenith represents left hand circular (LHC) and the nadir right hand circular (RHC) polarisation. All of the linear polarisation states lie around the equator. Elliptical polarisation states lie everywhere else. Two angles are required to define the point $\mathrm{P}$ on the sphere, $(\tau, \phi)$ or $(\alpha, \delta)$, the latter two being referred to as the Deschamps (or spinor) parameters and their values range accordingly:

- $\tau$ : Ellipticity angle $(-\pi / 4$ to $+\pi / 4),-\pi / 4$ (LHC), 0 (linear), $+\pi / 4$ (RHC).

- $\phi$ : Orientation (tilt) angle $(-\pi / 2$ to $+\pi / 2),(\tau, \phi)$

- $\quad \alpha$ : Auxiliary (or spinor) angle $(0$ to $+\pi / 2)$

- $\delta$ : Phase difference $(-\pi$ to $+\pi)$ between two orthogonal linear polarisations
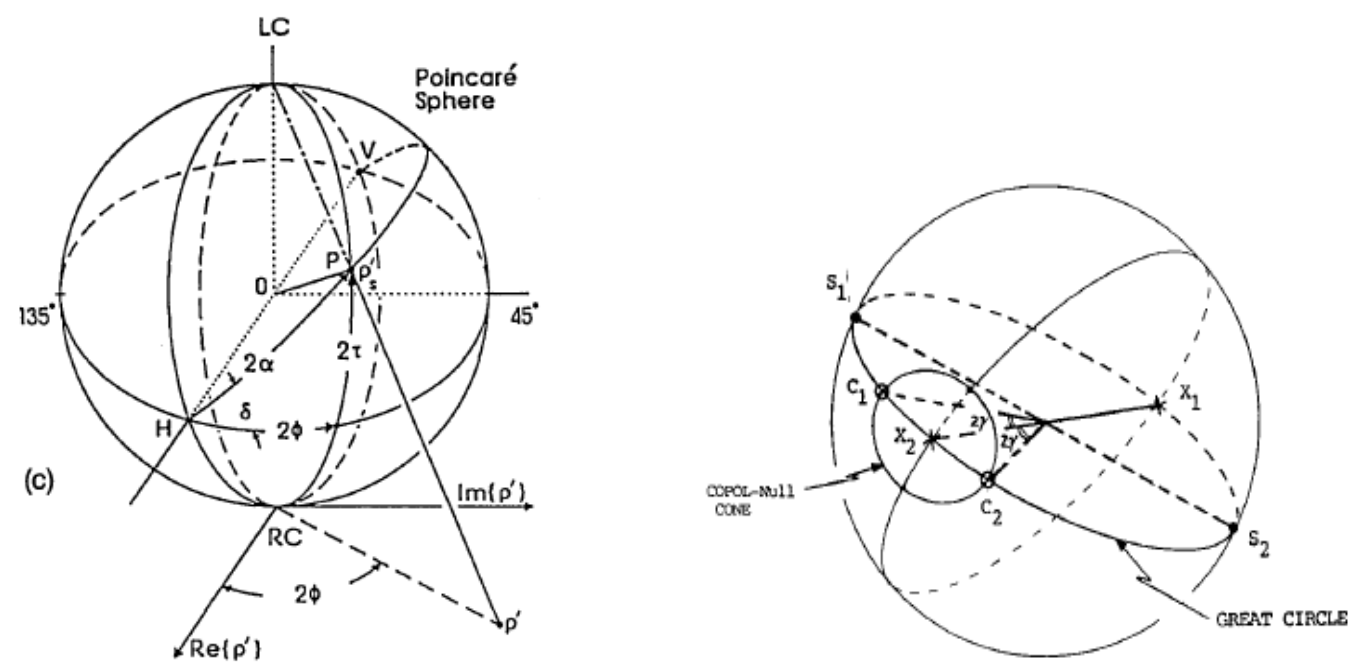

Figure 3 (a) Polarisation state on Poincaré sphere. [4] (b) Polarisation fork on Poincaré sphere. [5] 


\section{THE HUYNEN TARGET PARAMETERS}

The Huynen Fork target parameters represent the full (phase and amplitude) polarimetric response of a target, in an analogous way to how the Stokes Parameters characterise the general state of polarisation of radiation. The Huynen Fork has coordinates in the Poincaré Sphere. The main feature of the Huynen Fork is that the coordinate of the handle $\mathrm{X}_{1}$, indicates the polarisation which gives the maximum co-polar reflection and the minimum cross-polar reflection [6] as illustrated in Figure 3 from [5]. A sub-maximum, $\mathrm{X}_{2}$, also appears as the central prong in the Fork and is antipodal (180 opposite) to coordinate $X_{1}$. The maxima on the cross-polar responses have coordinates $S_{1}$ and $S_{2}$ and are antipodal, and together with coordinates $\mathrm{X}_{1}$ and $\mathrm{X}_{2}$, they form a great circle. Along this great circle lie the coordinates of the co-polar nulls, $\mathrm{C}_{1}$ and $\mathrm{C}_{2}$, which are symmetrical about the coordinate $\mathrm{X}_{2}$. The Huynen target parameters [7] consist of seven fundamental parameters (listed below) which are generated from the three complex numbers of the Sinclair matrix for a monostatic radar configuration. The existence of these coordinates in the Poincaré Sphere was first recognised by Kennaugh and developed more fully as a sensing modality by Huynen.

The Huynen Fork sometimes has only four coordinates shown, these being $\left(\mathrm{X}_{1}, \mathrm{X}_{2}\right)$ and $\left(\mathrm{C}_{1}, \mathrm{C}_{2}\right)$, in which case it is referred to as a three-pronged fork. Other times all six coordinates are shown, including $\left(\mathrm{S}_{1}, \mathrm{~S}_{2}\right)$, in which case it can be referred to as a five-pronged fork, in fact looking more like a tree than a fork.

The Huynen target parameters are derived [4] from the cross-polar null ( $\rho$-formulation) and are:

1. $\mathrm{m}=$ The maximum target size (measured at the co-polar maximum).

2. $\phi_{\mathrm{m}}=$ Target orientation (tilt) angle. $\phi(-\pi / 2$ to $+\pi / 2)$ about the view direction, measured in the horizontal plane of the Poincaré sphere.

3. $\tau_{\mathrm{m}}=$ Target Ellipticity angle: the angle of the co-polar maximum and Xpol null (X1) on the Poincaré sphere, ranging from $-\pi / 4$ to $+\pi / 4$. Some refer to this as the target helicity and is indicative of the degree of asymmetry in the target about the view direction; it being zero for symmetric targets.

4. $v=$ Target skip angle: $v(-\pi / 4$ to $+\pi / 4) 0$ for flat plate, $\pi / 4$ radians for dihedral, $\pi / 8$ radians for quarter waveplate and is related to the number of reflections from the target.

5. $\Upsilon=$ Target characteristic (fork) angle, varying from 0 to $\pi / 4$.

6. $\delta_{\mathrm{m}}=$ Phase of the polarisation ratio of the co-polar maximum varying from $-\pi$ to $+\pi$.

7. $\alpha_{\mathrm{m}}=$ Spinor angle, varying from 0 to $+\pi / 2$.

That are generated from just three parameters; $\rho_{x n 1}$ or $\rho_{x n 2}$ and $\lambda_{1}$ and $\lambda_{2}$

Cross Polar Null's in HV basis:

$$
\begin{aligned}
& \rho_{x n 1,2}=\frac{-B \pm \sqrt{ }\left(B^{2}-4 A C\right)}{2 A} \\
& A=S_{H H}{ }^{*} S_{H V}+S_{H V}{ }^{*} S_{V V} \\
& B=\left|S_{H H}\right|^{2}-\left|S_{V V}\right|^{2} \\
& C=-A^{*}
\end{aligned}
$$

Co Polar Null's in HV basis are given by: 


$$
\rho_{c n 1,2}=\frac{\left(-S_{H V} \pm \sqrt{ }\left(S_{H V}^{2}-S_{H H} S_{V V}\right)\right)}{S_{V V}}
$$

The co and cross-polar nulls presented in this paper are calculated in the linear (HV) basis but could easily be defined in another basis via the simple application of a unitary transformation.

Deschamps parameters for the co and cross polar nulls:

$$
\begin{aligned}
& \alpha_{n 1,2}=\tan ^{-1}\left(\left|\rho_{n 1,2}\right|\right) \\
& \left.\delta_{n 1,2}=\arg \left(\rho_{n 1,2}\right)\right)
\end{aligned}
$$

Phase of the unitary transformation matrix $[\mathrm{U}] \psi_{1}$ and $\psi_{4}$ are:

$$
\begin{aligned}
& \psi_{1}=-\frac{\delta_{x n 2}}{2}-\frac{\pi}{2} \\
& \psi_{4}=\frac{\delta_{x n 2}}{2}-\frac{\pi}{2}
\end{aligned}
$$

Orientation angle $\phi$ for the co and cross polar nulls are:

$$
2 \phi_{n 1,2}=\tan ^{-1}\left(\tan 2 \alpha_{n 1,2} \sin \delta_{n 1,2}\right)
$$

Ellipticity angles of co and cross polar nulls are:

$$
2 \tau_{1,2}=\sin ^{-1}\left[\sin \left(\delta_{n 1,2}\right) \sin \left(2 \alpha_{n 1,2}\right)\right]
$$

Unitary transformation matrix is:

$$
\left[\mathrm{U}\left(\rho_{x n 1}\right)\right]=\frac{1}{\sqrt{\left(1+\rho_{x n 1} \rho_{x n 1}{ }^{*}\right)}}\left[\begin{array}{cc}
1 & j \rho_{x n 1}{ }^{*} \\
\rho_{x n 1} & -j
\end{array}\right]
$$

Transformation of the Sinclair matrix in the new basis is:

$$
\left[S^{\prime}(A B)\right]=\left[U\left(\rho_{x n 1}\right)\right]^{T}[S]\left[U\left(\rho_{x n 1}\right)\right]
$$

Basis vector based on $\rho_{\mathrm{xn} 1}$ of the Sinclair (HV) matrix gives:

$$
\left[S^{\prime}(A B)\right]=\left[\begin{array}{cc}
\lambda_{11} & 0 \\
0 & \lambda_{22}
\end{array}\right]
$$

Target characteristic (fork) angle $\Upsilon$ is:

$$
\Upsilon=\frac{1}{2} \tan ^{-1} \sqrt{\frac{\left|\lambda_{22}\right|}{\left|\lambda_{11}\right|}}
$$

Target skip angle $v$ is:

$$
v=\frac{1}{4}\left(\arg \left(\lambda_{11}\right)-\arg \left(\lambda_{22}\right)\right)
$$


Target size $\mathrm{m}$ is:

$$
m=\left|\lambda_{11}\right|
$$

Target spinor parameter $\alpha_{\mathrm{m}}$ is:

$$
\alpha_{m}=\tan ^{-1}\left|\rho_{x n 1}\right|
$$

\section{MODELLING OF TARGET SINCLAIR MATRICES}

Modelling of target Sinclair matrix for a target is a good way to begin to understand how targets reflect (or scatter) coherent waves from a full polarimetric radar sensing scenario. A validated model has value in that it enables modelling of sophisticated targets and scenarios, which may be too costly to measure either in the laboratory or in-situ.

The simplest targets to begin modelling with are the canonical targets of the old-bounce (plane surface, sphere, trihedral) the even-bounce (the dihedral or roof-top), the dipole (straight wire or polariser) reflector and helix (right or left-handed). Considering the reflection these and disregarding any phase effects to due path lengths to the radar the Sinclair matrix for the reflectors are [8] for the old-bounce:

$$
S_{S S}=\left[\begin{array}{ll}
1 & 0 \\
0 & 1
\end{array}\right]
$$

the even-bounce with an edge at an angle $\delta$ to the horizontal (the second equality for $\delta=0$ ):

$$
S_{D S}=\left[\begin{array}{cc}
\cos 2 \delta & \sin 2 \delta \\
\sin 2 \delta 0 & -\cos 2 \delta
\end{array}\right]=\left[\begin{array}{cc}
1 & 0 \\
0 & -1
\end{array}\right]
$$

the straight wire (dipole) orientated at $\alpha$ to the horizontal (the second equality for $\alpha=0$ ):

$$
S_{\text {Dip }}=\left[\begin{array}{cc}
\cos ^{2} \alpha & \cos \alpha \sin \alpha \\
\cos \alpha \cdot \sin \alpha & \sin ^{2} \alpha
\end{array}\right]=\left[\begin{array}{ll}
1 & 0 \\
0 & 0
\end{array}\right]
$$

the helix, where \pm refers to the right or left-handedness of the helix:

$$
S_{\text {Helix }}=\left[\begin{array}{cc}
1 & \pm j \\
\pm j & -1
\end{array}\right]
$$

the quarter-wave plate [5], where $\mp$ refers to $+90^{\circ}$ phase advance in the vertical polarisation and the minus to a $90^{\circ}$ retardation in the phase:

$$
S_{\lambda / 4}=\left[\begin{array}{cc}
1 & 0 \\
0 & \mp j
\end{array}\right]
$$

Descriptions of other types of targets can be found in $\mathrm{PhD}$ dissertation from Huynen [7]. More complex targets may be described by using linear combinations of these basic targets.

Using the above Sinclair backscatter matrices for the canonical targets, the analysis above enables the Huynen target parameters to be determined for these targets and their coordinates shown in the Poincare sphere. For the single bounce plane reflector this is shown in Figure 4. A single solution for the Fork coordinates results from the calculation. However, the solutions for the co-polar maximum and the co-polar sub-maximum are multivalued, as the plane reflector has no orientation about the view direction; it is assumed to be circularly symmetrical. This can be shown in the solution by introducing a small amount of noise into the Sinclair matrix. Calculation of the Fork coordinates in this fashion then shows multi-valued solutions. However, the co-polar minima are always antipodal and at the top and bottom of the Poincaré Sphere, as a plane reflector will always flip circularly polarised radiation to its orthogonal orientation, ie right- 


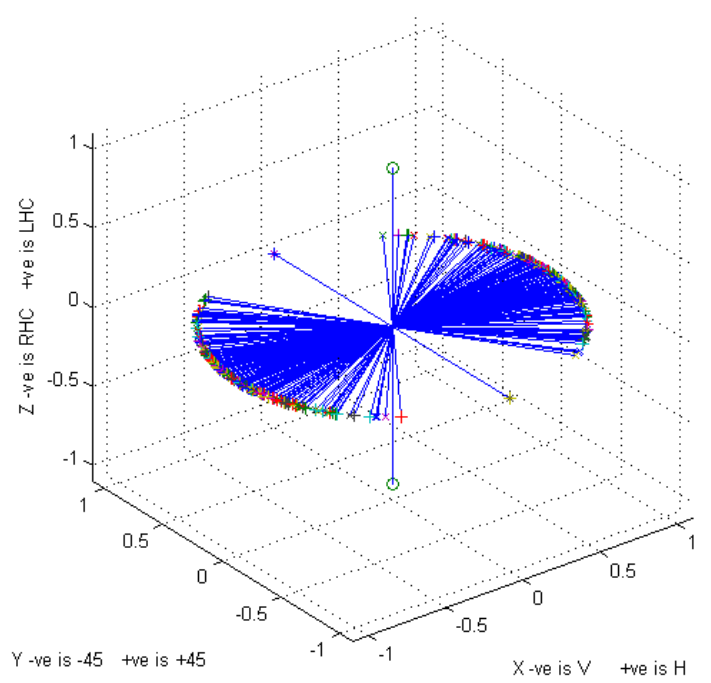

Figure 4. For a single bounce (plane) reflector the co-polar maxima ( $\mathrm{x})$ and sub-maxima $(+)$ are shown together with the co-polar nulls (o). The plane reflector has an infinite number of solutions for the co-polar maxima in the horizontal plane. hand circularly polarised radiation is flipped to the lefthand polarisation. The angles between the co-polar maxima and minima are always $90^{\circ}$, so the Fork characteristic angle $\gamma$ at half this value is always $45^{\circ}$ for the plane reflector.

For the dihedral reflector the coordinates of the Huynen Fork are shown in Figure 5. In a similar fashion the multivalued co-polar maxima are illustrated by introducing a small amount of noise into the Sinclair matrix. The copolar maximum and sub-maximum are anti-podal and are distributed along a great circle perpendicular to the horizontal mid-plane of the Poincaré Sphere. The orientation of this great circle and that of the co-polar nulls are directly related to the orientation of the dihedral. The angle between the co-polar maxima and nulls is always $90^{\circ}$, so the Fork characteristic angle $\gamma$ at half this value is always $45^{\circ}$ for the dihedral reflector.

The Huynen Fork coordinates for the dipole are shown in Figure 6. The Fork in this case is always in the mid-plane of the Poincare sphere and the position of the co-polar maximum indicates the dipole orientation. This can be seen in the figure when the orientation of the dipole changes from $0^{\circ}$, to $45^{\circ}$ and to $90^{\circ}$. Furthermore, the co-polar submaximum is co-located with the co-polar nulls, meaning the Fork characteristic angle $\gamma$ is zero.
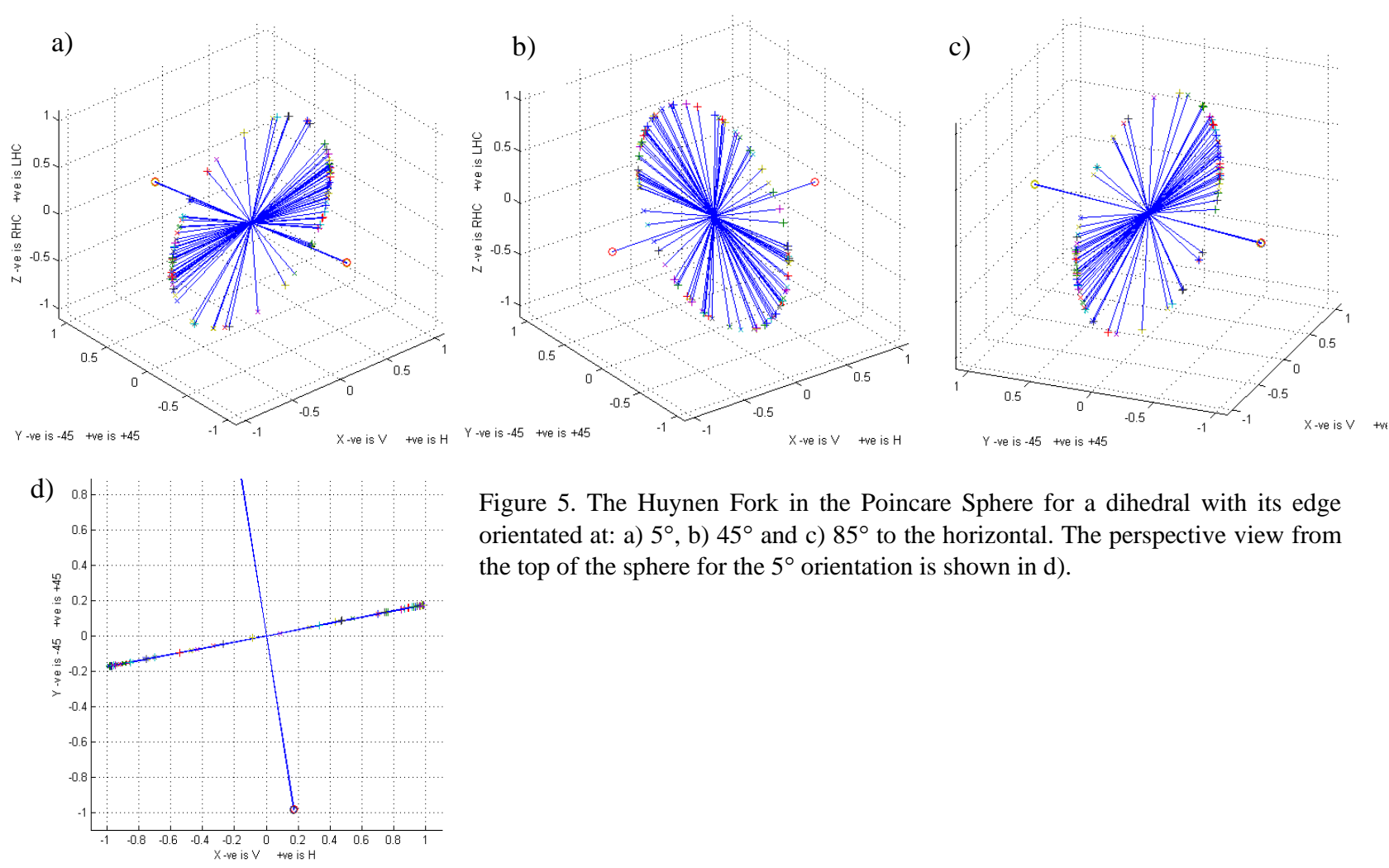

Figure 5. The Huynen Fork in the Poincare Sphere for a dihedral with its edge orientated at: a) $5^{\circ}$, b) $45^{\circ}$ and c) $85^{\circ}$ to the horizontal. The perspective view from the top of the sphere for the $5^{\circ}$ orientation is shown in $\mathrm{d}$ ). 

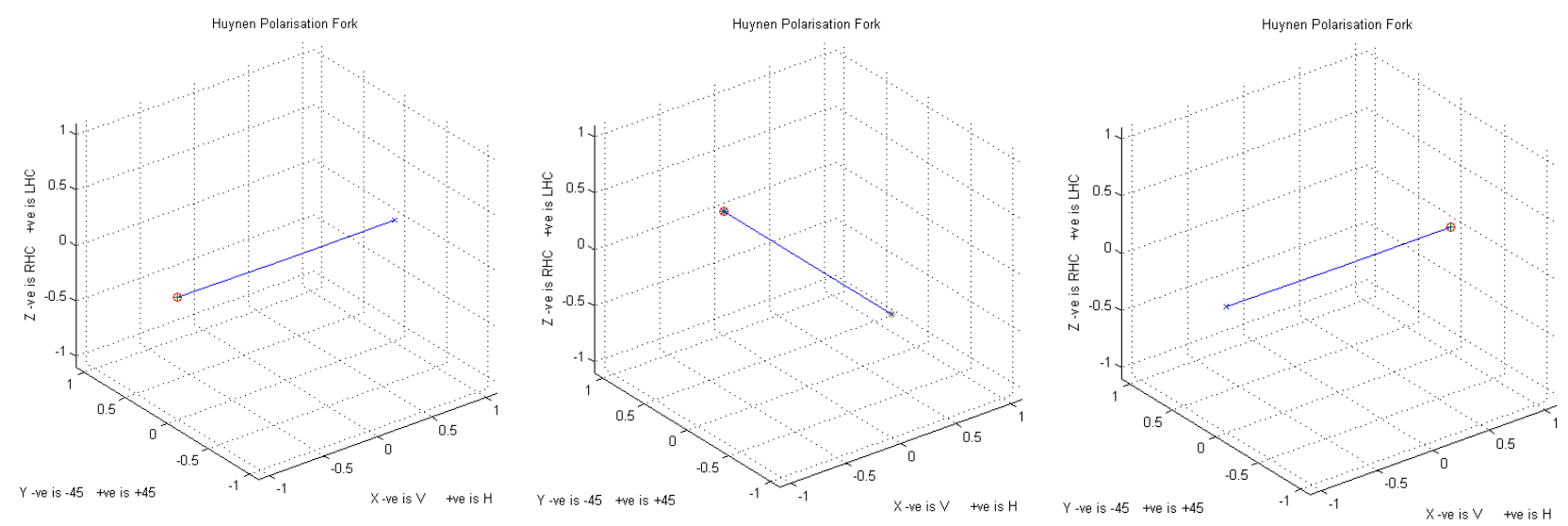

Figure 6. The Huynen Polarisation Fork for the dipole, orientated (from left to right) at $0^{\circ}, 45^{\circ}$ and $90^{\circ}$ to the horizontal. The copolar-maximum is represented by the $\mathrm{x}$ and the co-polar sub-maximum is represented by + and co-aligned with the co-polar nulls. The Fork angle $\gamma$ is zero.

The Huynen Fork coordinates for the right-handed and left-handed helixes are shown in Figure 7. They are single valued. The co-polar maximum indicates the maximum reflection is for circular polarisation and the co-polar nulls are antipodal to this, meaning the fork angle $\gamma$ is zero. This indicates maximum reflection when the circular polarisation is aligned with the handedness of the helix and a null in the reflections for the orthogonal circular polarisation.
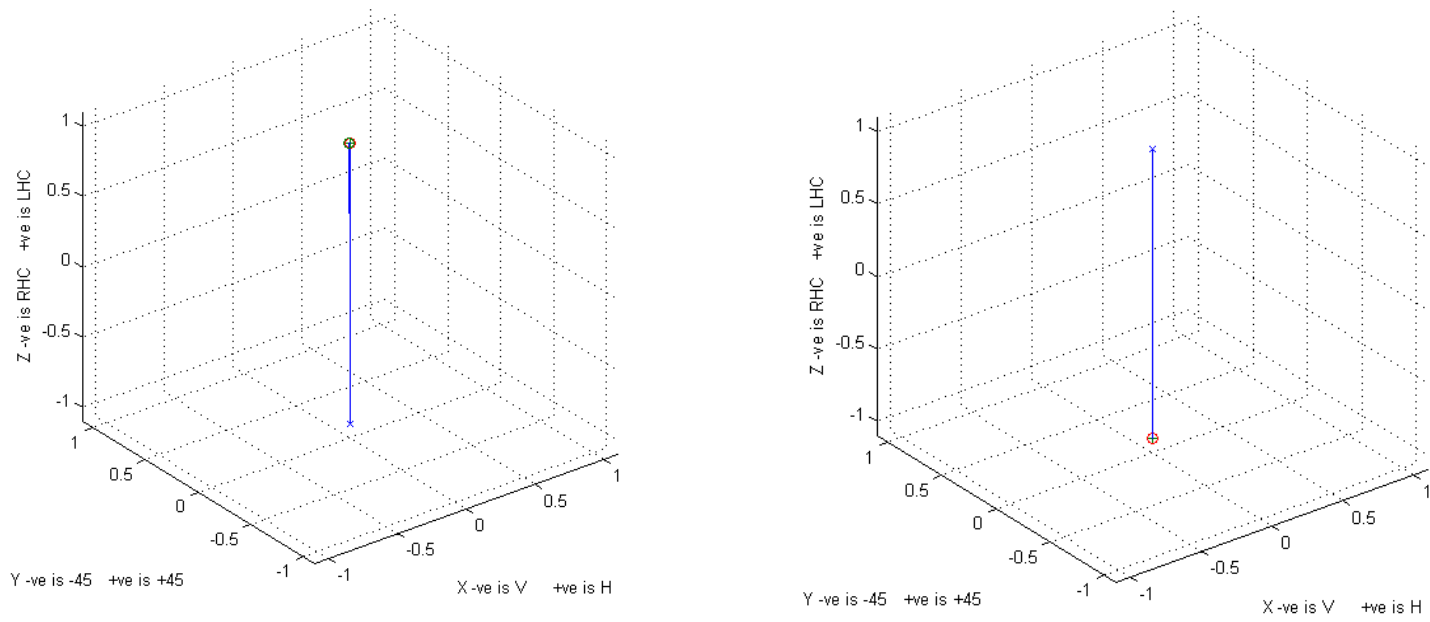

Figure 7. The Huynen Polarisation Fork for the lefthand (left) and righthand helixes. The co-polar sub-maxima are collocated with the co-polar nulls. 


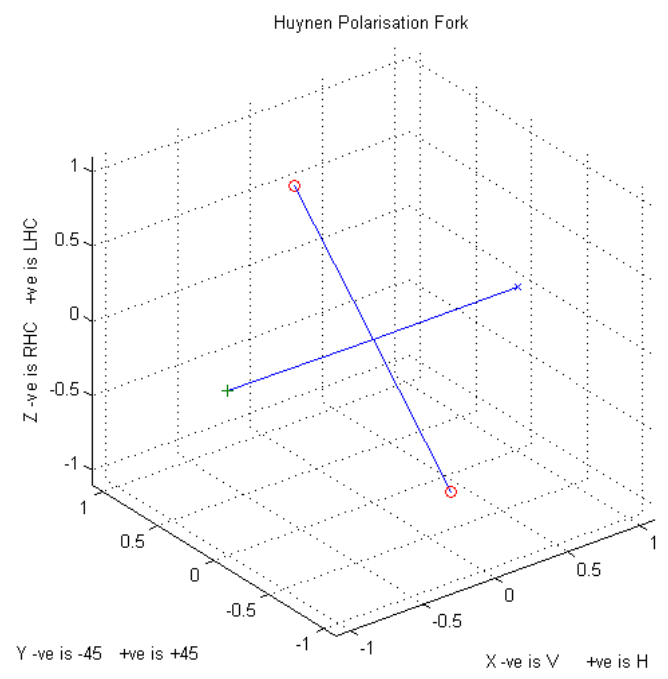

Figure 8. The Huynen Polarisation Fork for a quarterwave plate. Only a single value is shown here. The relative delay in one of the linear polarisations causes a shift of the co-polar nulls away from the zenith and nadir of the Poincare Sphere.

The Huynen Fork coordinates for a quarter-wave plate are shown in Figure 8.

The modelling of these canonical targets agrees well with the previous measurements presented in [1].

\section{MEASUREMENTS OF THE HUMAN TORSO}

Key to understanding the response of target in situ together with its background is in the understanding of how the target background looks. In most radar sensing scenarios the background generates a far greater radar return than the target. In that sense the background represents clutter. If the clutter can be well understood it enables the general strategy employed in radar processing to be exercised; that being the subtraction of the clutter from the measured signal so only the signature of the target remains. Algorithms can then process the target signature for the purposes of recognition.

Plots are presented in this paper of the Huynen polarization fork response of the torsos of two individuals and compared. The individuals were measured in two different positions, aligned with torso perpendicular to the boresight of the antenna and with the torso presented side on to the boresight. Measurements were taken with hands and arms above the head and at the side for each position.

For the measured results the cross-polar null $\mathrm{X}_{1}$ (the handle of the fork) is represented by blue (X)'s, the $\mathrm{X}_{2}$ nulls (central prong of the fork) are represented by red $(+)$ 's, the co-polar nulls $\left(\mathrm{C}_{1}\right.$ and $\left.\mathrm{C}_{2}\right)$ are shown as $(\mathrm{O})$ 's for the measured responses presented below.

Figure 9 shows the front of the human torso of two individuals aligned perpendicular to the antennas boresight and with the hands and arms positioned above the head. The left plot shows the torso for subject 1 whilst the right plot shows subject 2. The responses are similar to the response that is attained from a simple canonical radar target such as a flat plate or sphere Figure 9 (left and right). The flat plate and sphere has its cross-polar nulls located around the equator on the Poincaré sphere (as in the simulation of Figure 4) indicating that for the linear polarisations no change in orientation takes place upon reflection, for circular polarization conversion does take place. However unlike the flat plate or sphere where the cross-polar nulls are randomly distributed around the equator on the Poincare sphere it will be noted that some orientation information also seems to be present for both individuals. Orientation is indicated by the blue cross-polar nulls and appears to differ between individuals, the reason for this is not known at this moment but could be caused by a belt or zip on the individuals measured.

Figure 10 shows the front of the torso of the two subjects with torso aligned with the boresight of the antenna; however this time both individuals have their hands and arms positioned at the side of their torsos. From the plots both subject 1 and 2 are similar however this time the co-polar nulls are shifted away from the zenith and nadir axes and the cross-polar nulls are angled away from the equator of the Poincaré sphere. The Huynen Polarisation Fork is effectively being tilted. 
Interpretation of the tilt in the Fork may be attributed to the double reflection from the arms and the torso. This may be considered as making a contribution from a dihedral reflection. The effective reflection is therefore a linear combination of the single bounce reflection form the bulk or the torso, with a smaller amount of reflection caused by the dihedral response. The composite response is therefore a combination of the simulations shown in Figure 4 and Figure 5.

There may also be a contribution to the response shown in Figure 10 from relative delays of the horizontal and linear polarisations caused by the double reflection. From this respect the response may be like that of a waveplate. The wave plate also brings the co-polar nulls away from the zenith and nadir of the Poincare Sphere as indicated in Figure 8 and shown by the measurement illustrated in Figure 14.

Figure 11 shows the torso of both individuals but this time the torso is positioned with its side facing the boresight of the antenna and with arms and hands above the head. The response is similar that of the torso positioned perpendicular to the boresight as shown in Figure 9 and for that of the flat metal plate shown in Figure 13 (left) and Figure 14 (left). Very little helical conversion is visible for both individuals suggesting that only single odd bounce reflection is taking place.
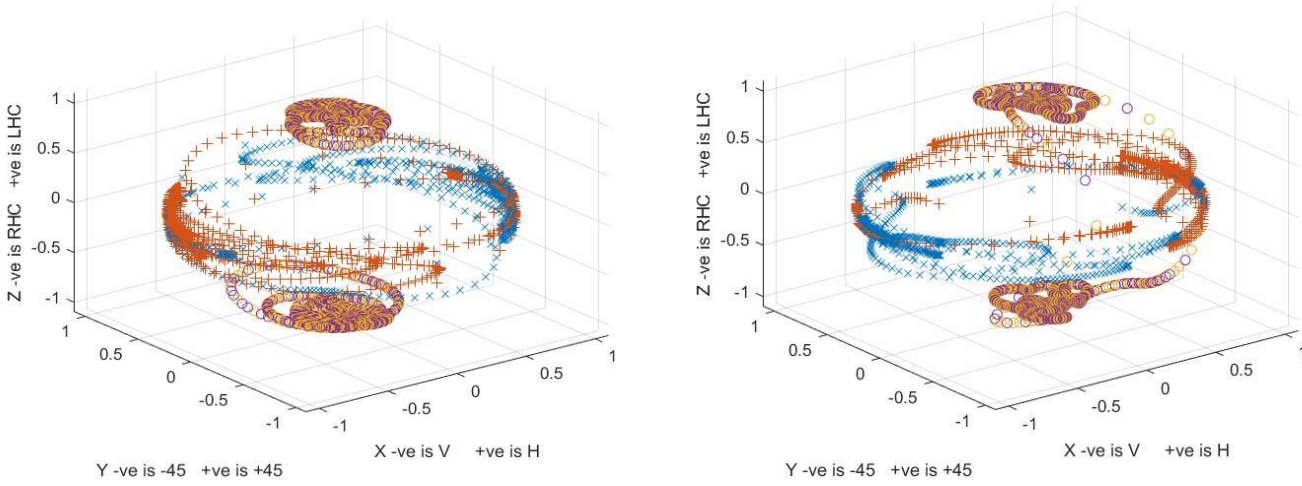

Figure 9. Human torso front-on with hands above head (subject 1 left, subject 2 right).
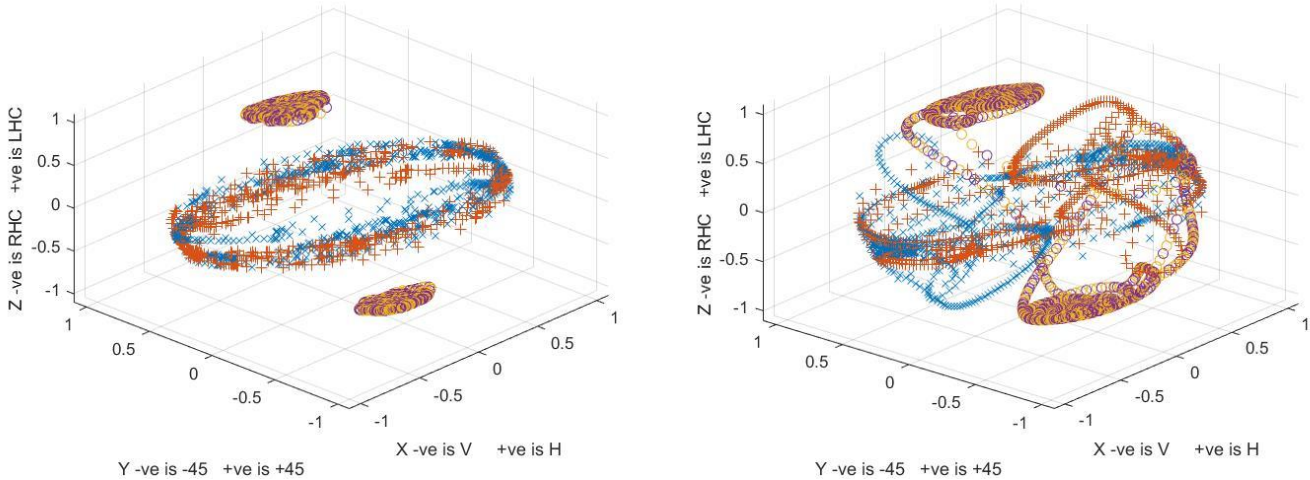

Figure 10. Human torso front-on with hands and arms at the side (subject 1 left, subject 2 right). 

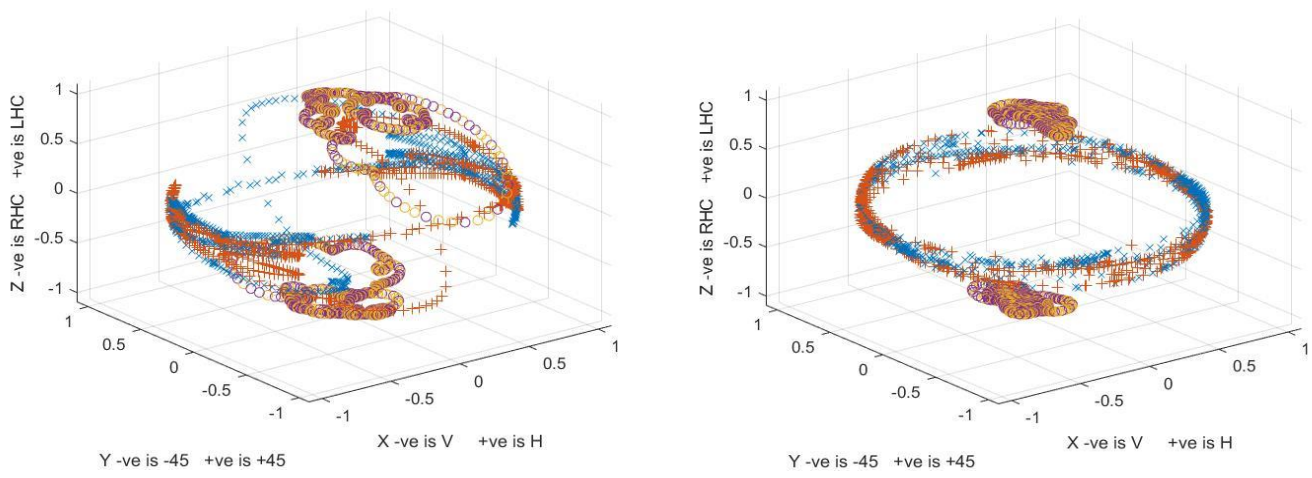

Figure 11. Human torso sideways-on with hands above head (subject 1 left, subject 2 right).

Figure 12 shows the torso for both individuals again presented side on to the boresight of the antenna however this time the arms and hands are at the side of the torso. The response of both individuals is again similar to that of a flat plate single (odd) bounce reflector as simulated in Figure 4.
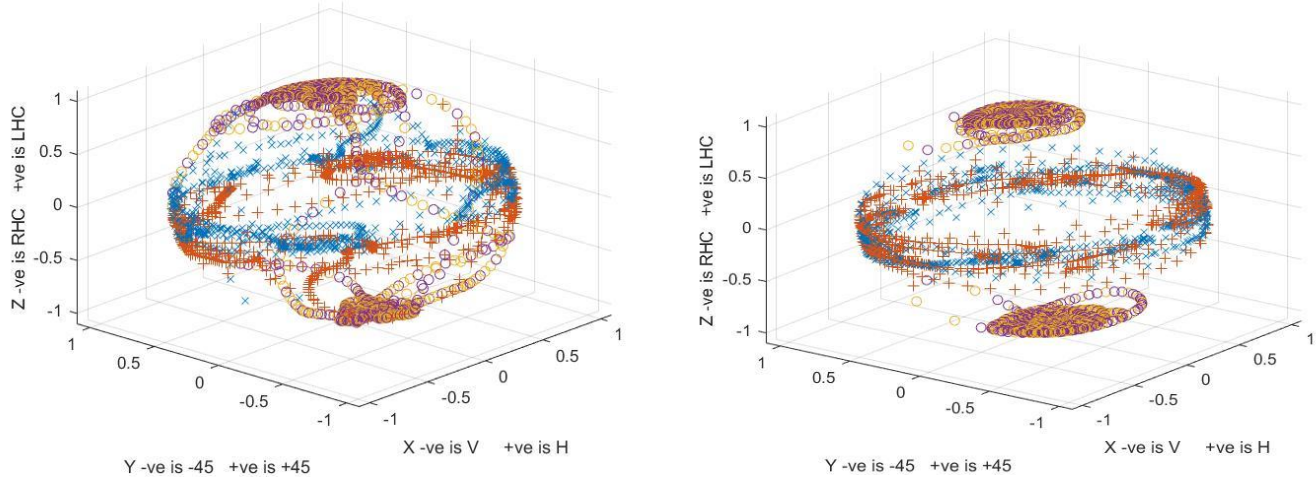

Figure 12. Human torso sideways-on with hands and arms at the side (subject 1 left, subject 2 right).

In some of the above plots of the coordinates of the Huynen Fork there appears to be deviation in the co-polar nulls $\left(\mathrm{C}_{1}\right.$, $\mathrm{C}_{2}$ ) away from the zenith and nadir and a shift of the co-polar maxima $\left(\mathrm{X}_{1}, \mathrm{X}_{2}\right)$ from the mid-plane of the Poincare Sphere. The coordinates shown in the plots represent 801 different frequencies over the $18 \mathrm{GHz}$ to $26 \mathrm{GHz}$ band. Since these points of deviation appear to trace out a locus, this suggests that there is structure over sections of this band where there is for some reason deviation from a simple plane surface reflector response. This might be caused by metal belt buckles or rings. Understanding of this phenomenon requires further investigation.

To show the effect of a waveplate, such a component was constructed from a plane metal sheet and a horizontal wire grid, these being illustrated in Figure 13. This component generates a greater delay in the vertical linear polarisation as this wave has to travel further before it is reflected. A comparison between the Huynen Polarisation Fork for these two cases is shown in Figure 14. This demonstrates indeed that the waveplate shifts the co-polar nulls from the zenith and nadir. 


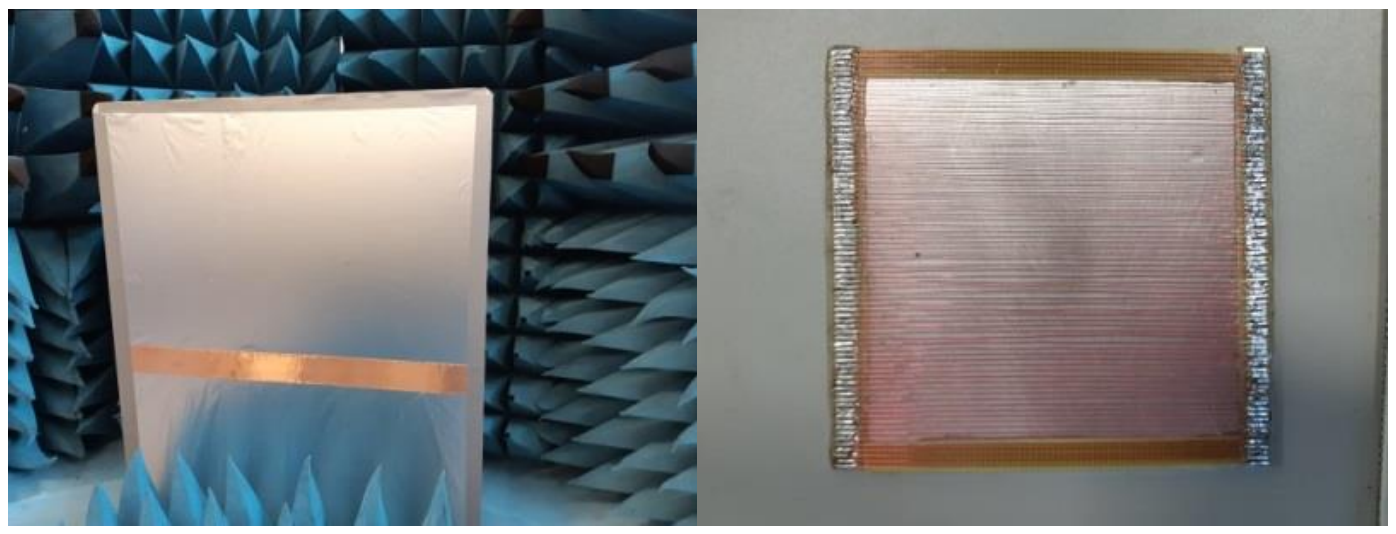

Figure 13 Flat metal plate (left), waveplate with wires horizontal (right).
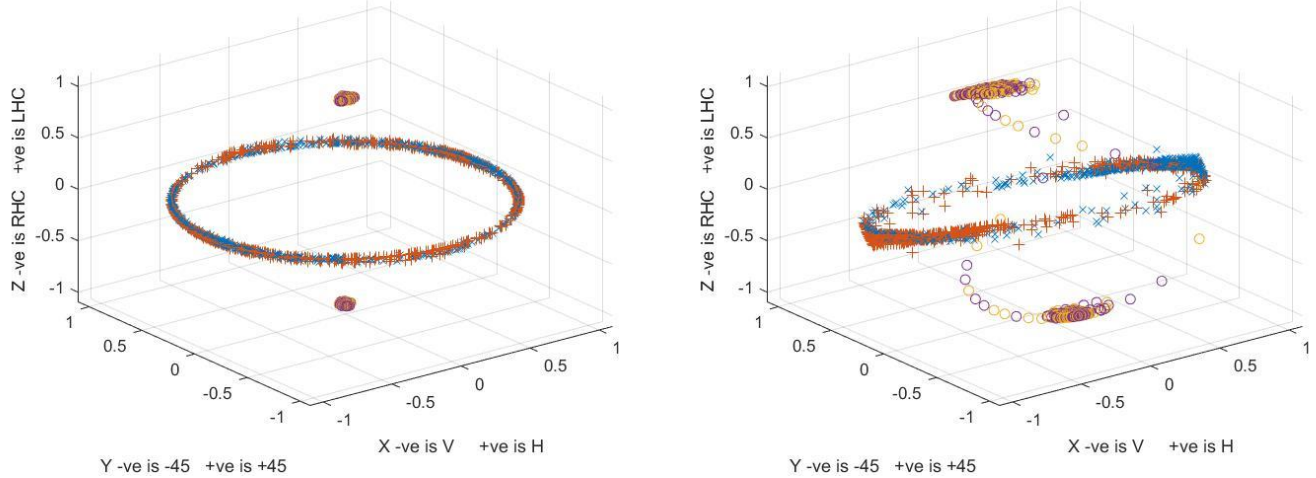

Figure 14 Flat plate reflector (left) waveplate with wires horizontal (right)

\section{CONCLUSION}

Simulations presented in this paper closely match the measurements of canonical radar targets such as flat plate/sphere, corner (dihedral), dipole and helical structures. The validity of simulations gives credibility to simulations of more complex targets. More complex targets could be simulated by combinations of a number of simpler targets that been simulated successfully. These models may be extended to simulate the human body.

For concealed weapons detection the response of the human torso represents unwanted clutter that makes detection of weapons and PBIED's more difficult. The torso aligned perpendicular to the beam produces a single bounce response similar to that of a flat plat or sphere, whilst with the arms and hands at the side produce a conversion in to helical polarization upon reflection. The torso presented side on to the beam produces a single bounce (flat plate type) refection regardless of if the arms are located at the torso side or above the head. The Huynen polarization fork responses presented in this paper of the torsos of two individuals show similar repeatable signatures for each of the positions presented to the beam of the radar. These signatures could be subtracted from measurements made of the human torso with weapons present, leaving the response of the weapons alone.

\section{FUTURE WORK}

Future work will be undertaken to identify the signatures of a variety of threat devices by themselves and when concealed under clothing on the human torso. Further decomposition algorithms, such as the H, $\alpha$ technique from Cloude will be applied [9] [10]. 


\section{REFERENCES}

[1] Blackhurst, E.; Salmon, N.; Southgate, M.;, "Experimental determination and simulations of the Huynen target parameters for full polarimetric millimetre wave concealed weapon recognition," in Proc. SPIE vol. 10800, Europe Security+Defence, Millimetre Wave and Terahertz Sensors and Technology XI", Berlin, 2018.

[2] Alessandro, Navarrini; L., Plambeck Richard, “A Turnstile Junction Waveguide Orthomode Transducer," IEEE TRANSACTIONS ON MICROWAVE THEORY AND TECHNIQUES, 2006.

[3] A. Xi and W. Boerner, "Determination of the characteristic polarization states of the radar target scattering matrix $[\mathrm{S}(\mathrm{AB})]$ for the coherent monostatic and reciprocal propagation space by using the complex polarisation ratio $\rho$ transformation formulation," Opt. Soc. Am. A, vol. 9, no. 3, pp. 437-455, 1992.

[4] Xi, A.Q.; Boerner, W.M.; "Determination of the characteristic polarization states of the radar target scattering matrix $[\mathrm{S}(\mathrm{AB})]$ for the coherent monostatic and reciprocal propagation space by using the complex polarisation ratio $\rho$ transformation formulation," Opt. Soc. Am. A, vol. 9, no. 3, pp. 437-455, 1992.

[5] Agrawal, A.P.; Boerner, W. M., "Redevelopment of Kennaugh's Target Characteristic Polarisation State Theory Using the Polarization Transformation Ratio Formalism for the Coherent Case," IEEE Transactions on Geoscience and Remote Sensing, vol. 27, no. 1, pp. 2-14, 1989.

[6] Boerner, W.M.; Yan, W.L.; Xi, A.Q., "On the Basic principles of Radar Polarimetry: Target Characteristic Polarisation State Theory of Kennaugh, Huynen's Polarization Fork Concept, and its Extension to the Partially Polarized Case," Proceedings of the IEEE, vol. 79, no. 10, pp. 1538-1550, 1991.

[7] Huynen, J.R, Phenomenological theory of radar targets, PhD dissertation, Drukkerij Bronder, Rotterdam, The Netherlands: Delft University of Technology, 1970.

[8] Massonnet, D.; Soyris, J-C, Imaging with synthetic aperture radar, Lausanne: EPFL Press, 2008.

[9] Keydel, E.H.W.; Pottier, E.; Hellmann, M.; Cloude, S.R.; Ferro-Famil, L., Radar Polarimetry and Interferometry, Brussels, Washington and Ottawa: NATO, 2004.

[10] Cloude, S.R.;, Polarisation - applications in remote sensing, Oxford University Press, 2010. 